\title{
MONITORING OVERWEIGHT AND OBESITY IN NSW: A GUIDE
}

Vicki Flood, Karen Webb and Ross Lazarus

Department of Public Health and Community Medicine

University of Sydney

Louise Baur

Department of Paediatrics and Child Health

University of Sydney

T his article describes why it is important to monitor overweight and obesity, and outlines some of the standard methods developed in the report Monitoring overweight and obesity in NSW', one component of the NSW Food and Nutrition Monitoring Plan². The report includes information on how to define and measure overweight and obesity, standards for classifying people as overweight or obese and options for obtaining weight status data on the NSW population.

\section{WHY IS IT IMPORTANT TO MONITOR OVERWEIGHT AND OBESITY IN NSW?}

Obesity is a risk factor for a range of chronic diseases, including non-insulin-dependent diabetes mellitus, coronary heart disease, hypertension, hyperlipidaemia and gall bladder disease ${ }^{3}$. Monitoring overweight and obesity provides information on its prevalence and trends. Monitoring can be used by policy makers and field workers to highlight an area of concern for intervention, and to assess the effectiveness of intervention. Overweight and obesity was identified as a high-priority issue for nutrition monitoring in consultations conducted as part of the NSW Food and Nutrition Monitoring Plan.

The 1994 NSW Health Promotion Survey estimated the prevalence of overweight and obesity in NSW, based on selfreported data, as 48 per cent for males and 31 per cent for females ${ }^{4}$. Reducing the prevalence of overweight and obesity is a goal within the overall strategy of reducing cardiovascular disease ${ }^{5,6}$.

Further, Area Health Services in NSW are increasingly focusing on population health. Performance agreements with the NSW Health Department include strategic activities and performance indicators for overweight and obesity.

\section{OUTLINE OF SOME RECOMMENDATIONS IN THE GUIDE}

To monitor overweight and obesity accurately, standard methods for the collection, measurement and analysis of data are required.

\section{What to measure}

For population monitoring, simple techniques are needed to indicate the prevalence and degree (mild, moderate, severe) of obesity (excess stores of body fat). However, there is no practical accurate measure of body fat for use in large population studies.

Measurements of weight and height are simple, unobtrusive and relatively inexpensive for the assessment of weight status, and relate reasonably well to risk of some diseases.

Body fat distribution is also an important predictor of obesity-related morbidity and mortality. Abdominal obesity is associated with coronary heart disease, stroke, noninsulin-dependent diabetes mellitus and high blood pressure $^{7}$. Waist circumference measurement is recommended as a simple way to determine abdominal obesity ${ }^{3,9}$.

Sociodemographic information is required to interpret anthropometric data and to monitor trends of risk in groups. The information should include data on age, ethnicity, number of years of residency in Australia, and socioeconomic status, including income, education and occupation.

Monitoring weight-related attitudes and practices may assist in planning more effective policies and programs to promote healthy weight control in the population and to reduce the prevalence of obesity. Topics suitable for the development of population-based questions include: self-perception of body weight; weight loss behaviour and intentions; weight goals; actions pursued for weight maintenance, weight loss or weight gain; and

barriers to healthy weight management.

How to measure overweight and obesity

Obtaining accurate measurements of weight, height, length (in infants) and abdominal and hip circumferences is difficult. Protocols for measurement are needed to ensure accurate standard measures, comparable within a survey (so different observers get the same result) and between surveys. The protocols are adapted from the World Health Organisation's recommended protocols ${ }^{10}$. Box 1 shows the recommended protocol for measurement of height.

Self-reported weights and heights

Self-reported weights and heights have been used as surrogate measures of actual weights and heights in surveys in Australia and NSW. The advantages of these

\section{BOX 1}

\section{HEIGHT MEASUREMENT}

- Use a vertical board with an attached metric rule and a movable horizontal headboard.

- Clients should be barefoot or in thin socks and wearing little clothing so that positioning of the body can be seen.

- The client should stand on a flat surface with weight distributed evenly on both feet, heels together and the head positioned so that the line of vision is perpendicular to the body.

- The arms should hang freely, and the head, back buttocks and heels should be in contact with the vertical board.

- If a person cannot stand straight in this position, only the buttocks and heels or head are in contact with the vertical board.

- Ask the client to inhale deeply and maintain a fully erect position.

- Move the headboard down to the top of the head so that the hair is compressed.

- Record height to the nearest $0.1 \mathrm{~cm}$.

- Record two measurements and if they differ by more than $0.5 \mathrm{~cm}$, then take a third measurement. 


\section{BOX 2}

RECOMMENDED QUESTIONS TO OBTAIN

SELF-REPORTED DATA

1. How tall are you without shoes?

centimetres

or

feet inches

2. How much do you weigh without clothes or shoes? kilograms

stone pounds

methods are that they can be included in self-administered questionnaires and used in telephone surveys. Box 2 shows the recommended questions. We know that the overweight tend to under-report their weights and over-report their heights ${ }^{11}$, and that body mass index based on self-reported weight and height tends to be underestimated as both men and women get older ${ }^{12}$, but it cannot be assumed that the error in one group at one time applies to all groups and remains similar over time.

The validity of self-reported weights and heights should be checked (by comparing them with measurements) on various population groups over time. The conditions in which subjects report their weights and heights may also affect accuracy: for example, self-reported data from a face-to-face interview are likely to be different from those of telephone surveys or self-administered questionnaires, where there is no expectation of assessment and where the participant cannot be viewed by the interviewer.

The NSW Health Survey, beginning in 1997, will use selfreported weights and heights as the method for monitoring overweight and obesity for Area Health Services, and a validation study (comparing telephone responses with measured heights and weights) will be conducted.

Standards for classifying people as overweight or obese Body mass index (BMI: weight/height in $\mathrm{kg} / \mathrm{m}^{2}$ ) is used to classify weights in the general population. Cut-off points for BMI have varied in the past, making it difficult to compare information between surveys ${ }^{13}$. The guide includes a review of surveys used in NSW over the past decade, and outlines differences between surveys ${ }^{1}$. Recently, standard cut-offs have been recommended by WHO, and the analysis of the National Nutrition Survey reflects these. For comparison with past surveys, two standards may be required to classify overweight and obesity. Box 3 shows a summary of these cut-offs.

Where to collect information about the weight status of the NSW population

Ideally, population health surveys, similar to the National Heart Foundation risk factor prevalence surveys (but with a representative sample for states) would be conducted

\section{BOX 3}

STANDARDS FOR CLASSIFYING OVERWEIGHT AND OBESITY IN THE GENERAL POPULATION

1. Commonly used categories for comparisons with past surveys are as 'underweight', 'acceptable weight', 'overweight' and 'obese'. Underweight

BMI $<20$

Acceptable weight BMI 20 to 25

Overweight BMI 25 to 30

Obese $\quad$ BMI $\geq 30$

2. To maintain consistency with the National Nutrition Survey, a further refined breakdown of categories is recommended (using a modified version of the latest recommendations by WHO).

Severe thinness

BMI $<6$ (WHO grade 3 thinnesss)

Moderate thinness MI 16 to 17 (WHO grade 2 thinness)

Mild thinness

MI 17 to 18.5 (WHO

grade 1 thinness)

Normal weight

(WHO normal range 18.5

to 24.99. Report two categories separately)

BMI 18.5 to 20

BMI 20 to 25

Overweight

BMI 25 to 30 (WHO grade 1 overweight)

Obese BMI $\geq 30$ (WHO grade 2 overweight, 30.0 to 39.99 , WHO grade 3 overweight $\geq 40.00$ )

3. Combine the 'thin' categories and 18.5 to $<20$ of the normal category to provide an 'underweight' category for comparisons with past surveys.

regularly, to track the changes in population weights and other risk factors. In practice, such surveys are unlikely to occur often because of the cost of bringing people to a centre for measurement. Therefore, the most feasible ways of obtaining information about trends in overweight and obesity are longitudinal studies of particular groups and other general health surveys.

We have identified the following surveys (and there may be others) as opportunities to obtain data about weights in the NSW population:

The National Nutrition Survey, 1995: includes measured weights, heights, and waist and hip circumferences of people aged two years and over $(\mathrm{n} \approx 2,800$ in NSW).

The NSW Health Survey: self-reported weight and height data of people aged 16 years and over $(\mathrm{n} \approx 17,000$ in NSW), repeated at predictable intervals.

Measurement of the weights, heights, and waist circumferences of a subsample of the respondents from the NSW Health Survey. 


\section{Monitoring overweight and obesity}

- Continued from page 47

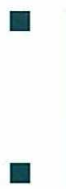

Women's Health Study: a longitudinal survey, 1996-2016; the baseline survey was conducted in 1996 on women aged 18-22 years, $45-49$ years and $70-74$ years $(n=12,000-15,000$ in each cohort).

Blue Mountains Eye Study: Stage 1, 1992-1994, Stage 2, 1997-1998; includes measured weights and heights on people aged 50 years and over $(n \approx 3,500)$.

Using a variety of surveys to obtain weight status information enables us to construct a picture of the weight status of various population groups. Use of the guide to plan or modify these surveys will ensure that standard methods are used for the collection and interpretation of weight status data.

\section{CONCLUSION}

Information alone is not sufficient to solve the problem of overweight and obesity. But the regular supply of information may continue to draw attention to a problem that requires more intensive and creative solutions than it has had in the past. Such information should lead to better planning, problem-solving and action. As plans for implementation of the National Obesity Strategy become more concrete ${ }^{7,14}$, NSW plans can be expanded.

1. Flood V, Webb K, Lazarus R, Baur L. Monitoring overweight and obesity in. NSW. Sydney: NSW Health Department, 1997.

2. Stickney B, Webb K Flood V, Hewitt M. The NSW Food and Nutrition

Monitoring Plan. Sydney: NSW Health Department, 1997. 3. Burton BT, Hirsh FJ, Van Itallie TB. Health implications of obesity: an NIH consensus development conference. Int J Obesity 1985; 9:155-59. 4. NSW Health Department. NSW Health Promotion Survey 1994. Technical Report. 1995.

5. Commonwealth Department of Human Services and Health. Better health outcomes for Australians, Canberra: AGPS, 1994

6. NSW Coronary Heart Disease Expert Working Group. Coronary Heart Disease Goals and Targets and. Strategies for Health Gain. Sydney: NSW Health Department, 1995.

7. National Health and Medical Research Council. Acting on Australia's weight: a strategy for the prevention of overweight and obesity. 1997.

8. Lean M, Han T, Morrison C. Waist circumference as a measure for indicating need for weight management $B M J 1995 ; 311(7): 158-161$.

9. Van der Tooy $\mathrm{K}$ Leenan $\mathrm{R}$, Seidell J, Deure

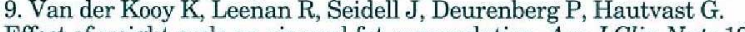

Effect of weight cycle on visceral fat accumulation. Am J Clin Nutr 1993; 58:853-7

10. World Health Organisation Expert Committee. Physical status: the use and interpretation of anthropometry. WHO Technical Report Series 854. Geneva: WHO 1995.

11. Rowland M. Self-reported weight and height. Am J Clin Nutr 1990; 52:1125-33.

12. Waters A. Assessment of self-reported height and weight and their use 12. Waters A. Assessment of self-reported height and weight and their use
in the determination of body mass index. Canberra: Australian Institute of Health and Welfare: 1993.

13. Harvey P, Marks G, Heywood P. Variations in estimates of overweight and obesity in Australia. Med J Aust 1991; 155:724.

\section{Guest editorial}

\section{Continued from page 41}

The second project concerned a more systematic approach to nutrition monitoring in NSW. While developing the catalogue was worthwhile, it became clear that there was a need for a coordinated approach if we were to furnish timely, relevant nutrition information to users: NSW Health Department planners, Area personnel and those in other agencies. The Department decided to develop a monitoring blueprint to complement the proposed national monitoring and surveillance plan and reflect the needs of the NSW Food and Nutrition Strategy, including the ability to monitor change among nutritionally vulnerable groups.

The articles in this issue report on the four main "products" of the second project. The first is an overview of the NSW Nutrition Monitoring Plan, which provides a blueprint for coordinated Statewide monitoring, and includes recommendations for collection, analysis and dissemination of timely and relevant nutrition information.

The second is a set of recommended short modules - that is, sets of questions or scales - for use in population-based surveys that lend themselves to the assessment of nutrition issues. These are interim recommendations, based on the best available information at the time of publication. They do not replace the need for continuing research and development, at the national level, into the best short modules for nutrition monitoring. Adoption of these interim recommendations by those who conduct population-based surveys in NSW will improve comparability of national, State and local survey data and lead to a clearer picture of progress towards State nutrition goals and targets.

The third product includes guidelines and recommendations for monitoring overweight and obesity. This is an important publication, given that the NSW Health Department has set targets for prevention and reduction of overweight and obesity, and for prevention of related conditions including cardiovascular disease, hypertension and high serum cholesterol levels. Data on overweight and obesity are needed so we can assess the effectiveness of interventions and promote the prevention of overweight and obesity. Most Area Health Service performance agreements with the NSW Health Department include strategic activities and performance indicators on diet, overweight and obesity, and physical activity. Standardised methods are needed for monitoring progress and demonstrating success in reducing the population prevalence of overweight and obesity.

The fourth product is a method for standardised monitoring of the food supplied by school canteens. This is required because of the considerable investment of the NSW Health Department in encouraging the availability of healthier foods in school canteens. It is important to be able to monitor the outcomes of this investment of public money.

1. Crowley S, Antioch K, Carter R, Waters A, Conway L, Mathers C. The cost of diet-related disease in Australia. Canberra: Australian Institute of Health and Welfare, 1992: 10-14.

2. Stickney B, Webb KL, Campbell C, Moore AR. Food and nutrition in NSW - a catalogue of data. State health pubn. no. (HP) 94-066. Sydney: NSW Health Department, 1994. ISBN 0731036581. 